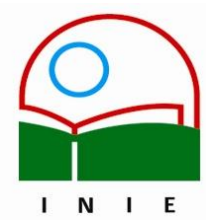

Actualidades Investigativas en Educación

Revista Electrónica publicada por el

Instituto de Investigación en Educación

Universidad de Costa Rica

ISSN 1409-4703

http://revista.inie.ucr.ac.cr

COSTA RICA

\title{
EL IMPACTO DEL DESARROLLO DE HABILIDADES SOCIO AFECTIVAS Y ÉTICAS EN LA ESCUELA \\ EL IMPACTO DEL DESARROLLO DE HABILIDADES SOCIO AFECTIVAS Y ÉTICAS \\ EN LA ESCUELA
}

Volumen 9, Número 3

pp. 1-21

Este número se publicó el 15 de diciembre 2009

María Isidora Mena Edwards

Claudia Romagnoli Espinosa

Ana María Valdés Mena

La revista está indexada en los directorios:

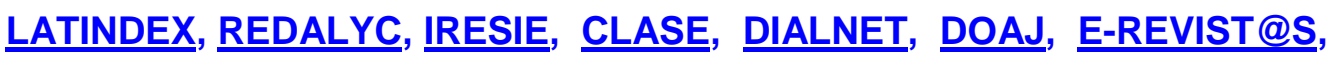

La revista está incluida en los sitios:

REDIE, RINACE, OEI, MAESTROTECA, PREAL, HUASCARAN, CLASCO

Los contenidos de este artículo están bajo una licencia Creative Commons

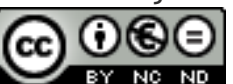




\title{
EL IMPACTO DEL DESARROLLO DE HABILIDADES SOCIO AFECTIVAS Y ÉTICAS EN LA ESCUELA EL IMPACTO DEL DESARROLLO DE HABILIDADES SOCIO AFECTIVAS Y ÉTICAS EN LA ESCUELA
}

\author{
María Isidora Mena Edwards ${ }^{1}$ \\ Claudia Romagnoli Espinosa ${ }^{2}$ \\ Ana María Valdés Mena ${ }^{3}$
}

\begin{abstract}
Resumen: El presente artículo analiza y sistematiza los resultados de investigaciones realizadas en Estados Unidos e Inglaterra sobre la aplicación de programas escolares de desarrollo socio afectivo. Después de describir las investigaciones, explica los ámbitos donde impacta este tipo de programas: mejora la disposición del contexto escolar para el aprendizaje, el apego a la escuela y la consecuente actitud disciplinada, la actitud y habilidad para aprender, el rendimiento académico, la salud mental y auto cuidado y naturalmente, las habilidades socioemocionales y ciudadanas.

Los resultados permiten relevar la importancia del desarrollo de la dimensión socio afectiva y ética al interior de la escuela pública chilena y latinoamericana, como un modo eficaz para enfrentar los desafíos de la educación del siglo XXI. Siguiendo las recomendaciones emanadas de las intervenciones exitosas, se sugiere no desvincular la dimensión social y emocional de la intelectual, resignificando esta doble dimensión de la escuela, que actúa como un círculo virtuoso.
\end{abstract}

Palabras clave: FORMACIÓN SOCIO AFECTIVA, FORMACIÓN ÉTICA, IMPACTO DE PROGRAMAS ESCOLARES, FORMACION DE HABILIDADES SOCIALES

\begin{abstract}
This article analyzes and systematizes the results of researches developed in The United States and England about the application of school programs of socio affective development. After describe the investigations, the article identifies the areas where this type of programs have impact: improvements of the school context for the learning, the attachment to the school and the consistent disciplined attitude, the willingness and skill to learn, the academic performance, the mental health and self care and naturally, the socio emotional and civil skills.

The results highlight the importance of development of the socio affective and ethical dimension inside Chilean and Latin-American public school, as an effective way to face educational challenges in the 21st century. Taking into consideration the recommendations coming from these successful interventions, it is suggested not to separate the social and emotional dimension from the intellectual one, re-meaning this double dimension of the school, which acts as a virtuous circle.
\end{abstract}

Key words: SOCIO AFFECTIVE LEARNING, ETHICAL LEARNING, IMPACT OF SCHOOL PROGRAMS, SOCIAL SKILLS LEARNING

\footnotetext{
${ }^{1}$ Doctor en Ciencias de la Educación, Pontificia Universidad Católica de Chile. Master en Formación de Valores, Universidad Virtual de Barcelona. Psicóloga, Pontificia Universidad Católica de Chile. Académica P. Universidad Católica de Chile, Escuela de Psicología. Directora General del Programa Valoras UC. Dirección electrónica: imenae@uc.cl

${ }^{2}$ Psicóloga, Pontificia Universidad Católica de Chile. Coordinadora General del Programa Valoras UC 2007. Dirección electrónica croma@uc.cl

${ }^{3}$ Psicóloga, Pontificia Universidad Católica de Chile. Profesional del equipo Programa Valoras UC. Dirección electrónica: amvaldem@uc.cl
}

Artículo recibido: 8 de enero, 2009

Aprobado: 30 de noviembre, 2009 


\section{Introducción}

La escuela ha sido tradicionalmente el lugar donde las sociedades forman a sus ciudadanos, entregando la socialización y el bagaje cultural que se requiere para ser partícipe en la vida social. También en Chile la legislación establece que la educación debe entregar posibilidades de desarrollar ambas dimensiones. Sin embargo, ha sido tradicional en la cultura escolar de nuestra educación pública, que la práctica se focalice en la transmisión académica y no en la socialización. La tendencia respecto de la formación social, afectiva y ética ha sido privilegiar un enfoque heterónomo, es decir aquel basado en el "deber ser" y en la obediencia a obligaciones establecidas externamente ${ }^{4}$, con muy poca sistematicidad y planificación. Salvo escasas y conocidas excepciones, los jóvenes terminan la vida escolar sin que la escuela se haya responsabilizado por su formación ética, afectiva y social.

La educación del siglo XXI hace un fuerte llamado a la educación pública para que se haga cargo de la formación socio afectiva y ética. La vida en una sociedad altamente tecnologizada, digitalizada, global, democrática, competitiva, y en constante transformación, requiere de un desarrollo humano en toda su potencialidad. Este desarrollo integral se necesita, en parte, para poder producir la vida social, política y económica y, en parte, para tolerar este estilo de vida. Ni el desarrollo económico y político del siglo XXI se logra sin desarrollo humano en toda su riqueza, dado el tipo de actividad, trabajo y labores que demanda, ni las personas pueden sobrevivir con dignidad la dinámica de la vida en esta sociedad moderna vertiginosa y potencialmente confundidora, si no se fortalece en su afectividad, sociabilidad y capacidad de discernimiento ético.

Sin embargo, en Chile, la demanda social por el desarrollo socio afectivo y ético no logra hacer gran mella a la cultura educacional tradicional de la educación pública. Una explicación a ello podría ser el costo de transformar la cultura escolar y capacitar a los profesionales de la educación en el nuevo enfoque. Pero es probable que el principal obstáculo sea que el enfoque de una socialización que forme para la autonomía, democracia, respeto en la diversidad, comunitarismo, sea demasiado antagónico con la cultura tradicional chilena que

\footnotetext{
${ }^{4}$ La heteronomía fue definida por Piaget (1932) como un estadio del desarrollo moral en que el niño acepta las consignas del adulto sean cuales sean las circunstancias en que estás se den ya que se entiende que el 'bien, lo bueno' es aquello concordante con lo que dice la autoridad, y el 'mal, lo malo' como aquello que se contrapone a ello.
} 
se reproduce en la escuela. Chile es un país con una cultura reconocidamente clasista, individualista y autoritaria. Esta cultura es la que corre por nuestras venas, y tiñe nuestras instituciones, nuestras prácticas de dirección, nuestros enfoques pedagógicos, estilos de relación y de discernimiento.

Hoy en Chile, pese a todos los esfuerzos e inversiones, hay bastante acuerdo en que nos está costando mucho reformar el ambiente educativo en pro de los objetivos formativos de la convivencia que se requiere en democracias modernas. También vamos lento en nuestros intentos de reformar el enfoque pedagógico hacia uno que permita a todos el logro de aprendizajes significativos.

Es nuestra convicción que si enfocamos la misión de la escuela en su doble dimensión de desarrollo humano y transmisión de saberes, y unimos a eso un cambio en la concepción del paradigma de relaciones y ambientes que forman, podremos avanzar no sólo en el desarrollo de las personas, sino también en aprendizajes y resultados en pruebas nacionales e internacionales. El camino no es fácil, porque el cambio cultural es muy complejo, y trasciende a la escuela. Significa asignarle a la escuela un rol más fuerte de transformación social/cultural, respecto del reproductor, cosa que tradicionalmente no es lo que la escuela hace. Sin embargo, tal como se han titulado varias iniciativas y publicaciones chilenas de la última década, ¿quién dice que no se puede?

En la búsqueda de caminos para voltear la cultura escolar en el sentido de la formación e incorporación de la dimensión socio afectiva y ética, se revisó, para el desarrollo de este artículo, una serie de investigaciones realizadas en Estados Unidos y en Inglaterra sobre efectos e impactos de programas escolares que apuntan a esta dimensión del crecimiento personal. A partir de esta revisión, se resumen los hallazgos de cinco grupos de investigadores, y se presenta, como núcleo de este trabajo, una organización de estos hallazgos en seis ámbitos de impacto, para demostrar que los programas escolares de habilidades socio afectivas son relevantes para la misión de la escuela. 


\section{Breve descripción de investigaciones sobre el impacto del desarrollo de habilidades socio afectivas}

Resumimos aquí los hallazgos de cinco grupos de investigadores que, en EEUU y en Inglaterra, han desarrollado meta análisis del efecto del desarrollo de habilidades socio afectivas.

\subsection{Durlak, Joseph; Weissberg, Roger; CASEL (2007). The impact of after- school programs that promote personal and social skills. Chicago, IL, EEUU}

El 2005 los autores realizaron un meta análisis que revisó 665 intervenciones educativas, que aplicaban programas de desarrollo de habilidades emocionales y sociales (SEL), programas de educación del carácter y programas de prevención. Este es el estudio más largo y rigurosamente científico que ha revisado las investigaciones sobre resultados de intervenciones que promueven el desarrollo socio emocional de los niños. La muestra incluyó intervenciones en escuelas, familias y comunidades designadas para promover habilidades personales y sociales en niños y adolescentes de entre 5 y 18 años, y se dividió en tres áreas:

- Intervenciones escolares que promovían aprendizaje socio emocional (SEL): revisión de 379 intervenciones.

- Programas fuera de la escuela

- Programas para las familias

Los resultados arrojaron un fuerte sustento empírico para validar los programas de desarrollo socio emocional, mostrando beneficios en el desarrollo de habilidades personales y sociales y en la reducción de problemas de conducta. Los dos primeros grupos, además, se relacionaron con mejoras sobre logros académicos.

En 2007, publicaron el meta análisis, basado únicamente en programas escolares que promovían el desarrollo de habilidades personales y sociales. Estos programas incluían el desarrollo de una o más habilidades de resolución de conflictos y problemas, autocontrol, liderazgo, toma de decisiones responsables, y mejoramiento de autoeficacia y autoestima. 
Se consideraron aquellos programas desarrollados durante todo el año escolar (septiembre a junio) y que ocurrían fuera del tiempo normal de colegio. El estudio incluyó también un grupo de control.

Se realizó una cuidadosa y sistemática búsqueda de estudios publicados y no publicados que proporcionaron información acerca de 73 programas. Con dicho material se condujo un meta análisis para evaluar la magnitud de los efectos que obtuvieron dichos programas.

2.2 El grupo de Greenberg, Mark; Weissberg, R.; O’Brien, M.; Zins, J.; Fredericks, L.; Resnik, H.; Elias, M. (2003), investigadores también de CASEL, en el departamento de psicología, de la Universidad de Illinois en Chicago

Su artículo llamado Enhancing school-based prevention and youth development through coordinated social, emotional, and academic learning, se encuentra dentro de las principales publicaciones de investigaciones acerca de la escuela que resumen múltiples estudios de escuelas SEL (de aprendizaje socio emocional). Consiste en una compilación de casos de escuelas basadas en prevención, que resume los resultados de los principales estudios de programas de prevención de aprendizaje socio emocional (SEL) y discute el papel de los programas de prevención en el contexto de las prácticas y políticas de otras escuelas y distritos.

\subsection{Berkowitz, Marvin; Bier, Melinda (2005), en la Universidad de Missouri, St.Louis, EE.UU.}

Los autores estudiaron distintas investigaciones que indagaban en el impacto de diversos "Programas de educación del carácter" implementados en la actualidad en EE.UU. De un centenar de estudios, seleccionaron por su rigurosidad científica a 78, los cuales a su vez, referían a 33 "Programas de educación del carácter" que mostraban alto grado de efectividad y eficacia, según los autores. Ellos analizaron y sintetizaron tanto los efectos diferenciales como los compartidos por los 33 programas de educación del carácter considerados 
efectivos, con el fin de construir una guía que sirva a la implementación de programas de educación en el futuro.

\subsection{Zins, Joseph; Bloodworth, M.; Weissberg, R., \& Walberg, H. (2004), del Teachers College, Columbia University, EEUU}

En un artículo titulado The foundations of social and emotional learning, este grupo revisa la relación entre el aprendizaje socio emocional (SEL) y el éxito escolar. La comprensión de este último factor, incluye la observación de componentes que van desde las calificaciones y resultados de pruebas estandarizadas, hasta actitudes, comportamientos y desempeño escolar.

\subsection{Department for Education and Skills (2005), en Inglaterra}

El estudio presentado por este grupo en el libro Excellence and Enjoyment: social and emotional aspects of learning, se centra en el uso de acercamientos de enseñanza y aprendizaje basados en el desarrollo de habilidades socio emocionales en los estudiantes y de desarrollo profesional continuo de todo el personal de la escuela. Su propósito fue entregar lineamientos para facilitar un acercamiento sistemático y espiral al aprendizaje.

\subsection{Carácter de los programas investigados}

Si bien no existe clara información publicada sobre el carácter de todos los programas investigados; resulta de ayuda consignar los elementos comunes de los datos disponibles a fin de propiciar una idea, general al menos, del tipo de intervenciones a los que se alude. Considerando las limitaciones de la información a la que se tuvo acceso, en este apartado sólo se hace referencia a dos de los grupos de investigadores enunciados; los cuales, sin embargo presentan claros elementos comunes que resulta esclarecedor consignar. 
Por una parte, CASEL $^{5}$ (CASEL a, 2007) explicita que en general los programas estudiados por ellos tendían a incorporar como ejes principales de acción la organización del ambiente para el aprendizaje junto a la formación intencionada y sistemática de ciertas habilidades socio afectivas. La descripción de CASEL (op cit) de los ejes, alude a la creación de un ambiente seguro y bien dirigido de aprendizaje y la instrucción secuenciada e intencionada en cinco áreas de competencias socio emocionales: conciencia de sí mismo (ej. reconocimiento de emociones, valores, intereses y habilidades personales); conciencia social (ej. habilidades de toma de perspectiva, empatía, búsqueda y uso de recursos para alcanzar sus metas); manejo de emociones y comportamientos (ej. expresión adecuada de emociones, manejo del estrés, control de impulsos, perseverancia para alcanzar metas personales y académicas); habilidades relacionales (para establecer y mantener relaciones sociales positivas, resistir la presión social inapropiada, buscar y dar ayuda, comunicarse efectivamente, negociar, resolver problemas); y toma de decisiones responsable (ej. considerando estándares éticos, normas sociales, el respeto a otros, consecuencias derivadas del actuar).

Berkowitz y Bier (2007) por su parte, notaron que de los 33 programas que analizaron, 27 se centraban en el currículum socio-emocional, que incluía comúnmente clases focalizadas en habilidades sociales (ej. habilidades de comunicación, audición activa, habilidades de razonamiento, asertividad, conciencia social); superación personal/autonomía y conciencia (ej. auto-control, técnicas de relajación, conciencia emocional, auto-conciencia); resolución de problemas y toma de decisiones.

Considerando las limitaciones de la información disponible, desconocemos si todos los programas estudiados comparten la misma estrategia de apuntar a habilidades académicas y de organización académica además de a programas socio afectivos. Tampoco hay certeza de que los programas socio afectivos sean similares. Pero es posible asumir la probabilidad de que haya bastante similitud, en tanto existe una tendencia mundial a lo que se llama una aproximación holística a la escuela, es decir, un abordaje que no separa el desarrollo de la escuela para formar tanto lo socio afectivo como para lograr aprendizajes de calidad.

${ }^{5}$ CASEL: Collaborative for Academic, Social and Emotional Learning Volumen 9, Número 3, Año 2009, ISSN 1409-4703 
Lo que sí coincide es que los investigadores afirman, a partir de sus resultados, que los beneficios de la formación socio afectiva no sólo son incuestionables, sino además muy variados y de amplio alcance.

Por último, Según Berkowitz y Bier (2007), las dimensiones más efectivamente afectadas, es decir, las que recibieron consistentemente el impacto más positivo (tuvieron los porcentajes más elevados de resultados positivos), son las siguientes:

1. Comportamiento sexual ( $91 \%, 10$ efectos significativos, de 11 testeados)

2. Conocimiento acerca del carácter $(87 \%, n=13$ de entre 15$)$

3. Cognición socio-moral $(74 \%, n=82$ de entre 111$)$

4. Habilidades de solución de problemas $(64 \%, n=54$ de entre 84$)$

5. Competencia emocional $(64 \%, n=31$ de entre 49$)$

6. Relaciones interpersonales $(62 \%, n=8$ de entre 13$)$

7. Ligazón a la escuela $(61 \%, n=19$, de entre 32$)$

8. Logros académicos (59\%, $n=31$ de entre 52$)$

9. Competencias comunicativas $(50 \%, n=6$ de entre 12$)$

10. Actitudes hacia los profesores $(50 \%, n=2$ de entre 4$)$

11. Violencia y agresión $(48 \%, n=50$ de entre 104)

12. Abuso de drogas $(48 \%, n=51$ de entre 104$)$

13. Moralidad personal $(48 \%, n=16$ de entre 33$)$

14. Conocimiento y actitudes hacia el riesgo ( $47 \%, n=35$ de entre 73$)$

15. Comportamiento escolar $(45 \%, \mathrm{n}=40$ de entre 88$)$

16. Comportamientos y actitudes pro-sociales $(43 \%, n=71$ de entre 167$)$

\section{3 Ámbitos de impacto en la misión de la escuela de los hallazgos de las investigaciones revisadas.}

A continuación, se organizan los impactos encontrados por las distintas investigaciones en seis ámbitos, que si bien tampoco está demostrado que sean ámbitos independientes entre sí, son significativos para demostrar que los programas escolares de habilidades socio afectivas son relevantes para la misión de la escuela, mirados desde distintas perspectivas. Cabe resaltar que las seis áreas de impacto no están descritas en orden jerárquico por significación estadística ni teórica. Se requieren más estudios para lograr algo así. 


\subsection{Ambiente escolar mejor y más solidariamente organizado para el aprendizaje.}

Las investigaciones muestran que el desarrollo de habilidades socio emocionales en los alumnos afecta significativamente el ambiente de aprendizaje de las escuelas, que se hace más cálido, seguro y protector. Los miembros de la comunidad escolar mejoran su capacidad de relacionarse entre sí, se hacen capaces de resolver pacíficamente los conflictos, y desarrollan mayor conciencia de las necesidades, intereses y emociones de los demás, apoyándoles y colaborando de mejor manera.

El estudio de Berkowitz y Bier (2005), que revisó la efectividad de 33 programas de educación del carácter (programas donde uno de los principales exponentes es Lickona, 1992) ${ }^{6}$ identificó, entre las dimensiones más efectivamente impactadas por estos estudios, el mejoramiento de las relaciones interpersonales (62\% de efectividad); de la actitud hacia los profesores (50\% de efectividad); la disminución de la violencia y agresión (45\% de efectividad); y entre otras, el aumento de comportamientos y actitudes pro sociales (43\% de efectividad); todas ellas, dimensiones que afectan el clima escolar.

La inclusión de niños que presentan dificultades específicas de aprendizaje se ha visto facilitada por la enseñanza de competencias emocionales y sociales. Los estudiantes con necesidades educativas especiales desarrollan habilidades para adaptarse a la clase y controlar su propio comportamiento, y los otros compañeros, más empatía, tolerancia y comprensión, así como actitudes y acciones pro sociales y solidarias (Rogers, 1994; Espstein and Elias, 1996; en Department for Education and Skills, 2005).

El estudio de Zins, Weissberg, Wang and Walberg (2004), realizó una compilación de casos para evaluar el impacto de los programas de desarrollo de habilidades socio emocionales y éticas en el mejoramiento de las actitudes, comportamientos y desempeños escolares. Encontraron que mejoraba la percepción de la escuela y el aula como ambientes seguros y ordenados; aumentaban las relaciones de cuidado entre estudiantes y profesores, lo que

\footnotetext{
${ }^{6}$ Para la National Comission on Character Education, la educación del carácter es cualquier iniciativa deliberada, en que el personal escolar, frecuentemente y en conjunción con los padres y miembros de la comunidad, ayudan a niños y jóvenes a convertirse en personas "cuidadoras", responsables y con principios (Williams \& Schnaps, 1999 en Vess and Halbur, 2003). 
fomenta el compromiso y contacto con la escuela; había una mayor cercanía con los profesores; una buena alianza con la familia para el apoyo del aprendizaje y comportamiento positivo de los alumnos; se promovía un aprendizaje cooperativo y manejo proactivo de la clase; y las normas comunicaban altas expectativas de desempeño académico.

Se ha visto que la generación de un buen ambiente, producto del desarrollo de las habilidades socio afectivas de los estudiantes, tiene efecto también sobre los docentes. Una investigación de Bryk \& Schneider (2002 en CASEL a, 2007), mostró que las escuelas caracterizadas por relaciones de mucha confianza tienden a tener más profesores que experimentan con prácticas innovadoras de enseñanza y que buscan mayor contacto con los apoderados. Esto es un factor que influye en que los alumnos asistan regularmente a la escuela y en que pongan mayor esfuerzo cuando se enfrentan con tareas difíciles.

El equipo del Department for Education and Skills (2005), en Inglaterra, enfatiza que el aprendizaje se construye en un contexto social, definido por un tipo de interacciones y las emociones que ellas provocan. Los procesos emocionales y sociales, inseparables de los procesos cognitivos, influyen en la relación del estudiante con el aprendizaje. Los programas de desarrollo socio afectivo promoveen de relaciones positivas, que producen emociones agradables, -como sentimiento de bienestar, sentirse seguro y valorado, alegría, la curiosidad, entusiasmo-, generando contextos estimulantes del aprendizaje y del compromiso con el aprendizaje (Greenhalgh, 1994; Weissberg and Elias, 1993; Silvestre, 1995; Perry, 1996; en Department for Education and Skills, 2005)

En la misma línea, contextos que promueven emociones desagradables como la tristeza, rabia, ansiedad y estrés, pueden bloquear el aprendizaje y contribuir a la emergencia de comportamientos que perjudican el aprendizaje, como agresividad, desesperanza. Tal como ha planteado Daniel Goleman (1997), el autor que relevó el concepto y la importancia de la inteligencia emocional, los estudiantes que están ansiosos, enojados o deprimidos, no aprenden; las personas que están en estos estados emocionales no pueden manejar eficientemente la información.

El mismo estudio afirma que un ambiente favorable de aprendizaje no sólo favorece el aprendizaje académico, sino todo aprendizaje cognitivo-social-emocional-comportamental- 
valórico. Por ejemplo, se ha estudiado que en atmósferas de comprensión, los alumnos se sienten más cómodos para acercarse e interactuar con profesores y pares, y de ese modo, fortalecen sus habilidades relacionales, entre otras (op.cit). Se ha visto que estudiantes que logran vínculos emocionales positivos con pares y con adultos que valoran el aprendizaje y tienen altas expectativas sobre su desempeño académico, toman una actitud hacia lo académico más positiva y se muestran más motivados por aprender (CASEL a, 2007).

\subsection{Actitudes y habilidades para aprender}

Zins, Weissberg, Wang and Walberg (2004), encontró fuertes impactos del desarrollo de habilidades socio emocionales y éticas sobre las habilidades de aprendizaje de los alumnos. Encontraron que los alumnos reportaron tener más conciencia de sus habilidades de aprendizaje, junto con tener más seguridad frente a ellas; se esforzaban más y estaban más intrínsecamente motivados para aprender; y fueron capaces de definir metas, manejar su estrés y organizar el abordaje de su trabajo de mejor manera. Adicionalmente, encontraron que los estudiantes comenzaron a tomar decisiones más responsables acerca de su estudio, comenzaron a finalizar sus tareas, y lograron superar mejor sus obstáculos de aprendizaje.

El meta análisis de Durlak, Weissberg y CASEL (CASEL a, 2007) comenta que el estudio de Linacres et al (2005) mostró que los estudiantes que reciben entrenamiento en habilidades cognitivas, sociales y emocionales, reportaron mayor auto eficacia y puntuaron más alto en la valoración de los docentes en cuanto a su atención y concentración, en relación a los estudiantes comparativos.

Mayer y Salovey (1997, en Department for Education and Skills, 2005), agregan que el desarrollo emocional ayuda además a priorizar, decidir, anticipar y planificar, todas competencias esenciales en el proceso de aprendizaje. Así también, se ha visto que el desarrollo de habilidades socio emocionales y éticas, promueve una comprensión más profunda de las materias tratadas en clases. Un estudio de Murdock (1999) citado, revisado por el meta análisis de Durlak, Weissberg y CASEL, reveló que aquellos adolescentes que caracterizaron su ambiente de aprendizaje como protector, con profesores y pares respetuosos que se ayudan unos a otros, es más probable que participen en clases y terminen sus tareas. 
Junto con ello, algunas investigaciones muestran que las habilidades socioemocionales pueden aumentar significativamente los aprendizajes cuando están integradas en diferentes áreas de contenido académico. CASEL (CASEL a, 2007) refiere que se ha observado que el desarrollo de habilidades de resolución de problemas favorecía la comprensión y análisis de eventos históricos. El estudio de Elías (2004, en CASEL a, 2007) señala que, por ejemplo, cuando a los estudiantes se les pide el uso de habilidades como la toma de perspectiva y la solución de problemas para comprender y analizar los acontecimientos históricos en una clase de artes del lenguaje, el aprendizaje en estas áreas de contenido mejora.

En esta dirección, se ha demostrado que la integración de lo social y emocional en el proceso de instrucción académica es un modo particularmente eficaz de promover competencias tanto socio-emocionales como académicas (Elías, 2004, en CASEL a, 2007).

\subsection{Mejoramiento del rendimiento académico y éxito escolar}

Múltiples estudios muestran cómo los programas mencionados no sólo impactan positivamente sobre los aprendizajes, sino también sobre los resultados académicos.

El meta análisis de Durlak, Weissberg y CASEL (CASEL a, 2007) relata que en Chicago, Joe Durlak de la Universidad de Loyola y Roger Weissberg de la Universidad de Illinois (2005) revisaron estudios sobre 379 programas escolares que aplicaron programas socio afectivos y éticos. Encontraron una mejora significativa en el rendimiento académico de los niños en pruebas estandarizadas. Apoyando este hallazgo, el meta análisis cita las investigaciones de Rimm-Kaufman (2006); Flay \& Allred (2003), Flay, Allred, \& Ordway (2001).

El mismo meta análisis expone una investigación de Dymnicki (2006, en CASEL b, 2007), en 43 escuelas con programas de desarrollo de habilidades socioemocionales y éticas, donde las puntuaciones en las evaluaciones académicas mejoraron en un 14\% (14 ptos. percentil). A lo anterior se suman los hallazgos del estudio de Berkowitz y Bier (2005), que mostró que los programas de educación del carácter presentaban un 59\% de efectividad en la mejora de los logros académicos. 
Así también, una evaluación longitudinal de un programa social de resolución de conflictos, encontró que los estudiantes que participaron tuvieron en conjunto mayores logros académicos seis años después que el grupo comparativo. Además, observaron que los alumnos de aquellos profesores que implementaron más completamente el programa, se desempeñaron mejor en lenguaje, arte y matemáticas que los del grupo comparativo (Elias, Gara, Schuyer, Branden-Muller, \& Sayette, 1991; en CASEL a, 2007).

El meta análisis de Durlak, Weissberg y CASEL también muestra que estudios longitudinales de programas preescolares que fomentaban las competencias socio emocionales documentaron numerosos resultados positivos para los participantes, incluyendo la necesidad de una menor cantidad de tiempo asignado a programas de educación especial, mayores índices de alfabetización y de graduación de la escuela secundaria (Schweinhart, Barnes \& Weikart, 1993; Schweinhart \& Weikart, 1997; en CASEL b 2007).

La relación entre habilidades socio afectivas y éticas y el rendimiento también ha sido apoyada por investigaciones que han intentado definir las características de los alumnos exitosos. Se ha estudiado que los alumnos exitosos tienen interacciones sociales e intelectuales activas con sus pares y profesores, participan activamente en el aprendizaje (en contraposición con una recepción pasiva del conocimiento), son capaces de comunicarse efectivamente y de preguntar y pedir ayuda cuando lo necesitan, y son capaces de trabajar bien en grupos de aprendizaje cooperativo; todas ellas son competencias relacionadas con habilidades socioemocionales, éticas y comportamentales (CASEL a, 2000-2007).

Es un círculo virtuoso: mientras el desarrollo socio afectivo influye positivamente el rendimiento, el mejoramiento del desempeño académico a su vez es un factor que previene la emergencia de gran variedad de comportamientos de riesgo (Magín \& Loeber, 1996) y de problemas de salud mental (Symons, Cinelli, James, \& Groff, 1997; Blue, Beuhring \& Rinehard, 2000), ambos citados por el meta análisis de Durlak, Weissberg y CASEL..

Los estudios sobre "inteligencia emocional" (mirada más amplia del concepto de inteligencia que integra aspectos personales y sociales), han determinado que las competencias emocionales y sociales han mostrado ser más determinantes que las competencias cognitivas, para el éxito personal, académico y profesional (Goleman, 1997). 


\subsection{Desarrollo de un buen apego con la escuela, y la consecuente actitud disciplinada.}

El meta análisis de Durlak, Weissberg, y CASEL (CASEL a, 2007), cita varios hallazgos que muestran el apego a la escuela que producen los programas de desarrollo de habilidades sociales y emocionales: el meta análisis de Durlak y Weissberg (2005) que encontró el efecto de apego y actitud positiva hacia la escuela; el estudio de Battistich, Solomon, Watson, Schaps y Lewis (1997) señala que estos programas aumentan la sensación de comunidad escolar entre los estudiantes y el compromiso hacia la escuela; la investigación de Zins, et al (2003) muestra mejoramiento de actitudes hacia la escuela, junto con una mejora del comportamiento y desempeño en el colegio.

El mismo meta análisis de Durlak, Weissberg y CASEL refiere múltiples estudios que fundamentan cómo un buen apego hacia la escuela, a su vez, tiene un fuerte impacto sobre otras dimensiones. Entre ellas, se hace referencia al mejoramiento de la asistencia a clases por parte de los alumnos (Elias, Gara, Schuyer, Branden-Muller, \& Sayette, 1991); el mejoramiento del rendimiento académico general y en pruebas estandarizadas (Osterman, 2000); la disminución de comportamientos de alto riesgo (Resnick et al, 1997; Hawkins, Catalano, \& Millar, 1992); la presencia de niveles más bajos de distress emocional (Resnick et al, 1997).

Por otra parte, un meta análisis reciente de estudios que evaluaron cerca de 70 programas de desarrollo de estas habilidades (en niños de todos los niveles de la educación escolar), mostró una disminución de un $44 \%$ de suspensiones, y de un $27 \%$ con respecto a otras acciones relacionadas con disciplina. Los autores señalaron que esto se debía a que los niños desarrollaban mayor apego hacia la escuela, al mismo tiempo que desarrollaban habilidades básicas para tomar opciones saludables y éticas, resistir las influencias sociales negativas y manejar sus emociones productivamente (Durlak, Weissberg y CASEL, 2007).

El impacto en disciplina es muy comprensible teóricamente, en tanto ella se puede entender como la disposición y habilidad para acatar el rol que una comunidad le asigna, por el interés que se tiene, ya sea en pertenecer a esa comunidad o bien, -o además-, por el interés en las metas que esta comunidad permite conseguir. De ahí que se pueda entender esta correlación entre apego a la escuela y disciplina. Naturalmente, desarrollar apego tendrá Volumen 9, Número 3, Año 2009, ISSN 1409-4703 
impacto en la disciplina, en tanto el apego promoverá el querer ser parte de ese grupo y eventualmente también, valorar sus metas, siempre y cuando sean percibidas como justas y se cuente con las habilidades para respetarlas.

Apoyando este hallazgo, Twemlow y su equipo (2001 en CASEL a, 2007) también encontraron una reducción significativa de los índices de problemas de disciplina tras una experiencia en que se integró el desarrollo de habilidades socio emocionales y éticas en el currículo académico. Naturalmente, también mejoraron las calificaciones de los estudiantes en pruebas estandarizadas. Así también Dymnicki (2006 en CASEL b, 2007), en su estudio de 43 escuelas con programas de desarrollo de habilidades socio emocionales y éticas, mostró que se produjo una disminución significativa de suspensiones y expulsiones, un aumento de la asistencia, y un mejoramiento de la actitud de los alumnos hacia la escuela.

En la misma línea, Birch \& Ladd (1997 en CASEL b, 2007) mostraron que, por el contrario, cuando hay altos grados de conflicto entre profesor y alumno, los estudiantes presentan elevados niveles de evitación a la escuela, disminución del gusto/afecto por ella, menor autocontrol del comportamiento, y menores niveles de cooperación.

\subsection{Salud mental y conductas de riesgo}

Estudios longitudinales muestran que el desarrollo de competencias emocionales, sociales y de bienestar personal pueden reducir, en los jóvenes y en sus profesores, problemas de salud mental, tales como depresión, ansiedad, suicidio, desórdenes de la alimentación, estrés (Department for Education and Skills, 2005).

Así también el estudio de Resnick y su equipo (1997 en CASEL a, 2007), citado anteriormente, muestra cómo la generación de un clima escolar de cercanía, se correlaciona con una disminución de los niveles de distress emocional, pensamientos y comportamientos suicidas, violencia, abuso de sustancias y actividad sexual temprana, en los adolescentes evaluados.

El impacto demostrado en disminución de conductas de riesgo, que preocupan a la sociedad, se constituyen en medidas indirectas de promoción de salud (Zins, Bloodworth, 
Weissberg y Walberg, 2004). La habilidad para tomar buenas decisiones y opciones ayuda a los niños y adolescentes a evitar sentirse atraídos por comportamientos de alto riesgo y, como contraparte, los incentiva a participar en actividades favorables para su desarrollo positivo (CASEL a, 2007).

El desarrollo de habilidades socio afectivas ha demostrado tener un impacto estadísticamente significativo sobre las conductas de riesgo más relevantes que se detectan en los establecimientos educacionales, es decir, aquellas que impactan directamente el aprovechamiento de la escuela. Ellas refieren, en primer lugar, a la inasistencia y la deserción, y un poco más indirectamente, al consumo indebido de alcohol y drogas, la conducta sexual temprana, el embarazo, comportamientos antisociales y delincuencia (Zins, Bloodworth, Weissberg, \& Walberg, 2004; y Wilson, Gottfredson y Najaka, 2001, en CASEL a, $2007^{7}$.

En particular, una revisión de investigaciones de escuelas con programas de prevención para el abuso de sustancias y de violencia, mostró que la instrucción y práctica de competencias sociales y emocionales resultó ser un elemento crítico para el éxito de estos programas (Dusenbury, \& Falco, 1995; Dusenbury, Falco, Lake, Brannigan, \& Bosworth, 1997; en CASEL a, 2007). Esta correlación, la apoyan también Battitich, Schaps, Watson, Solomon y Lewis (2000; en CASEL a, 2007), quienes en un estudio encontraron una significativa disminución de los niveles de uso de drogas (junto con disminución de la delincuencia) en alumnos que habían participado de programas de desarrollo de habilidades socio emocionales y éticas.

En relación al comportamiento sexual, el estudio de Berkowitz y Bier (2005), ya citado, identificó esta dimensión como una de las más efectivamente afectadas por estos programas ( $91 \%$ de efectividad).

7 Este último grupo de investigadores desarrolló un meta análisis acerca de 165 estudios de programas de desarrollo de habilidades socio emocionales y éticas. Los hallazgos son casi por completo concordantes con el metanálisis de Zons, Bloodworth, Weissberg \& Walberg; sólo difieren en que este último grupo no reportan impacto significativo sobre la conducta sexual temprana y embarazo. 
Junto con lo anterior, el estudio citado revela el impacto de estos programas sobre otras conductas que afectan el ambiente escolar, como la violencia, el matonaje y la delincuencia (Zins, Bloodworth, Weissberg, \& Walberg, 2004); conductas que naturalmente son altamente perjudiciales para los mismos estudiantes. En esta línea, Linacres y su equipo (2005 en CASEL a, 2007) reportaron que los profesores estudiados puntuaron a aquellos estudiantes que recibían entrenamiento en habilidades cognitivas, sociales y emocionales con niveles más bajos de comportamiento agresivo, en relación a los estudiantes comparativos. También los calificaron con puntajes más altos en relación a sus habilidades de resolución de conflictos y habilidades prosociales (resultados coherentes con los reportados en otros estudios e intervenciones, tales como el de Flay y Allred, 2003, en CASEL a, 2007).

La importancia de estos hallazgos, cobra mayor fuerza considerando que se ha demostrado que la reducción de los comportamientos de riesgo favorece los aprendizajes y la posibilidad de éxito escolar. Una revisión de estudios y reportes sobre comportamientos de riesgo para la salud, encontró que los riesgos de salud, así como los daños intencionales, uso de sustancias, comportamiento sexual riesgoso, y pobre salud psíquica de los estudiantes, estaban directa y negativamente relacionados con los resultados educativos, comportamiento escolar, y actitud de estos en relación a la educación (Symons, Cinelli, James, \& Groff, 1997 en CASEL a, 2007). A su vez, un pobre desempeño académico es un factor que predispone a la emergencia de una gran variedad de comportamientos de alto riesgo, en contraposición con los logros académicos, que actúan como fuertes factores protectores frente a situaciones de riesgo, tal como fue mostrado anteriormente. Así también, la correlación especialmente fuerte en relación a la deserción e inasistencia que reveló este estudio, es importante en cuanto ellas son factores centrales en términos de éxito escolar (Zins, Bloodworth, Weissberg y Walberg, 2004).

De esta manera, las conductas de riesgo presentarían una elevada correlación con las posibilidades de logro de aprendizaje; su sinergia entonces puede traducirse en un potencial círculo vicioso, o en su prevención, uno virtuoso. 


\subsection{En los objetivos mismos de los programas: el mejoramiento de habilidades socio emocionales básicas y las habilidades ciudadanas.}

Se ha estudiado que los niños y jóvenes que han asistido a estos programas de desarrollo de habilidades, en efecto logran ser más auto conscientes (capacidad de reconocer sus emociones, describir sus intereses y valores, y valorar en forma certera sus fortalezas; ello, por ende afecta, a su sentido de confianza en sí mismos y en la esperanza hacia el futuro) y conscientes de los demás (capacidad de tomar distintas perspectivas y de empatizar con otros, reconociendo similitudes y diferencias individuales y grupales); manejan mejor sus emociones y comportamientos; presentan más habilidades para relacionarse con otros (pueden establecer y mantener relaciones sanas y gratificantes basadas en la cooperación, resistir la presión social inapropiada; prevenir, manejar y resolver constructivamente conflictos interpersonales; y buscar y dar ayuda cuando se necesita); y demuestran tomar decisiones más responsables (Department for Education and Skills, 2005).

El estudio de Berkowitz y Bier (2005), demostró que estos programas tenían un $64 \%$ de efectividad en el desarrollo significativo de habilidades de resolución de problemas.

Evaluaciones de programas de no violencia, de promoción de convivencia prosocial y de formación valórica confirman que educar en esta dirección es una manera de favorecer la formación de ciudadanos más comunicativos, más participativos y comprometidos, que confían y respetan a quienes los rodean y que tienden menos a la violencia. Estos estudios además señalan que el efecto de estos programas no sólo se limita al entorno escolar en que fueron aplicados, sino que se amplía también a contextos familiares, principalmente a las relaciones con los padres.

Una investigación de Johnson y Johnson (2000 en CASEL a, 2007), reportó que los estudiantes evaluados, una vez que terminaron su entrenamiento en resolución de conflictos, retuvieron sus conocimientos y habilidades de resolución de problemas a lo largo de los años escolares, fueron capaces de aplicar sus habilidades en los conflictos que ocurrieron en la sala de clase, y las transfirieron a instancias fuera del aula y de la escuela. 


\section{Conclusiones}

Los resultados de las investigaciones son claros: Los impactos de trabajar en la formación socio afectiva y ética son amplios y enormemente significativos.

En particular, resulta de gran relevancia el efecto que se ha mostrado que estos programas tienen sobre el aprendizaje. Buena noticia para la realidad chilena donde las políticas se han centrado tradicionalmente en el mejoramiento de éstos últimos muy por sobre la formación socio afectiva y ética. La visibilización de esta relación permitirá ir abriendo espacio a la necesidad y relevancia de organizar la convivencia escolar y formar en estas habilidades, junto con enfatizar la importancia de integrar ambos ámbitos para el logro de los objetivos escolares.

Este efecto estos programas sobre los aprendizajes está lejos de ser inesperado; todos ellos se orientan al aprendizaje e intencionan la integración de lo socio afectivo y ético en vistas del mejoramiento de él. Se entiende que el desarrollo de estas habilidades tiene sentido en la escuela en su relación directa y de mutua influencia con el objetivo del aprendizaje (reconocido como el centro del quehacer educativo). Un mejor ambiente de estudio y trabajo junto con el desarrollo de habilidades socio afectivas y éticas que posibilitan la convivencia esperada es lo que permitirá a los y las estudiantes sacar más provecho de su experiencia escolar; ayudará a los docentes a cumplir mejor con sus objetivos de enseñanza en un contexto más favorable y coordinado; potenciará la posibilidad de las familias de apoyar la educación de sus hijos. Lo socio afectivo y ético no puede ser desvinculado del aprendizaje, de lo contrario, la escuela no estaría cumpliendo con su misión.

Sin duda el tipo de las intervenciones descritas son posibles en escuelas mínimamente organizadas para resultar efectivas. De ahí el que sea un desafío mayor en nuestro país, donde gran parte de nuestras escuelas suelen presentar dificultades básicas para gestionarse. La necesidad de lograr escuelas mejor organizadas vuelve a ponerse en el tapete como prioridad educativa y los lineamientos que se entregan desde los programas de gestión de la convivencia escolar entregan elementos centrales para su mejoramiento.

Conviene también tener presente que las intervenciones en esta línea son multimodales, sus estrategias se dirigen a distintos focos y dimensiones del quehacer y organización escolar. Volumen 9, Número 3, Año 2009, ISSN 1409-4703 
Esta propiedad, si bien es costosa, es probablemente lo que las vuelve efectivas: acciones aisladas se ha visto que suelen tener efectos muy reducidos al encontrarse con enormes resistencias en el resto del sistema que sigue funcionando de acuerdo a su cultura previa. En Chile esto supone un nuevo desafío considerando la tendencia a realizar acciones separadas y desintegradas, producto, en parte de nuestra cultura burocrática. Ello no sólo reduce la eficacia sino además produce un elevado desgaste profesional. Los renombrados 'transversales' deben mostrar su transversalidad en la práctica, organización y vida escolar y no sólo en la declaración de intenciones... ello requiere una gestión capaz de abrir espacio a intervenciones sinérgicas que permitan contrarrestar la tendencia al status quo.

Por último, constituye un desafío el desarrollar en Chile y Latinoamérica un estudio que permita evaluar en esta región las intervenciones escolares que integren el desarrollo de habilidades socio afectivas y éticas a lo académico para evaluar los efectos que, sobre nuestra realidad y cultura, tienen estas acciones.

Mientras se realicen estos estudios, cabe apoyarnos en las investigaciones internacionales que con datos cuantitativos de fuerte significación empírica demuestran los efectos de estos programas sobre el aprendizaje, rendimiento, apego escolar, disciplina, prevención de riesgo, salud mental, desarrollo integral y formación de ciudadanía democrática. Ello permite arriesgarse, creer e invertir tiempo escolar en esta dirección, sabiendo que el modo pedagógico amable, democrático y dialogante, así como los ambientes que forman para la autonomía que promueven los programas de habilidades socio afectivas y éticas, ejercen gran impacto sobre múltiples dimensiones del quehacer educativo.

Atrevernos a generar políticas educativas serias en la organización de la convivencia escolar y la formación socio afectiva y ética permitirá no sólo la democratización de una educación de calidad para todos, sino además formar en las habilidades que requieren los ciudadanos para vivir y construir la democracia.

\section{REFERENCIAS}

Berkowitz, Marvin; Bier, Melinda. (2005). What Works in Character Education. A researchdriven guide for educators. CEP (Character Education Partnership), Universidad de 
Missouri,-St.Louis, EE.UU. Recuperado el 15 de marzo 2007 de www.rucharacter.org/file/practitioners 518.pdf .

CASEL (a) (Collaborative for Academic, Social and Emotional Learning). (2007). Benefits of SEL: SEL and Academics. Recuperado el 15 de marzo 2007, de www.casel.org/sel/academics.php

CASEL (b) (Collaborative for Academic, Social and Emotional Learning). (2007). Benefits of SEL: Meta-analysis. Recuperado el 15 de marzo 2007, de http://www.casel.org/sel/meta.php

Department for Education and Skills. (2005). Excellence and Enjoyment: Social and Emotional Aspects of Learning: Guidance. Primary National Strategy, Inglaterra. Recuperado el 15 de marzo 2007, de http://publications.teachernet.gov.uk/eOrderingDownload/DFES0110200MIG2122.pdf

Durlak, Joseph; Weissberg, Roger; CASEL. (2007). The Impact of After-School Programs that Promote Personal and Social Skills. Chicago, IL, EEUU. Recuperado el 20 de mayo 2007, de http:// www.casel.org/downloads/ASP-exec.pdf

Goleman, Daniel. (1997). La inteligencia emocional. Bantan Books, NY, Estados Unidos.

Greenberg, Mark.; Weissberg, Roger; O’Brien, Mary; Zins, Joseph; Fredericks, Linda; Resnik, Hank; Elias, Maurice. (2003). Enhancing School-Based Prevention and Youth Development through Coordinated Social, Emotional, and Academic Learning. En American Psychologist, 58 (6/7), 466-474. Recuperado el 15 de marzo 2007, de http://www.casel.org/downloads/AmericanPsychologist2003.pdf)

Lickona, Thomas. (1992). Educating for Character: How Our Schools can Teach Respect and Responsibility. Batan Books, New York, USA.

Piaget, Jean. (1932). El criterio moral en el niño. Traducción de "Le jugement moral chez l'enfant" (1974), Ed. Fontanella, Barcelona.

Vess, Kimberly, Halbur, Duane. (2003). Character Education: What Counselor Educators Need To Know. ERIC Clearinghouse on Counseling and Student Services Greensboro NC. Recuperado el 29 de julio de 2008, de http://www.ericdigests.org/2003$\underline{5 / \text { character.htm }}$

Zins, Joseph; Bloodworth, Michelle; Weissberg, Roger; Walberg, Herbert. (2004). The Foundations of Social and Emotional Learning. En Building Academic Success on Social and Emotional Learning: What Does the Research Say? (pp.3-22). Teachers College, Universidad de Columbia, NY, USA. Recuperado el 15 de marzo 2007 de http://www.casel.org/downloads/T3053c01.pdf)

Zins, Joseph; Weissberg, Roger; Wang, Margaret; Walberg, Herbert. (Eds). (2004). Building Academic Success on Social and Emotional Learning: What Does the Research Say? Teachers College Press, Columbia University, New York, USA. 